\title{
Thermal Equilibrium and Statistical Thermometers in Special Relativity
}

\author{
David Cubero, ${ }^{1}$ Jesús Casado-Pascual, ${ }^{1}$ Jörn Dunkel, ${ }^{2}$ Peter Talkner, ${ }^{2}$ and Peter Hänggi ${ }^{2}$ \\ ${ }^{1}$ Física Teórica, Universidad de Sevilla, Apartado de Correos 1065, Sevilla 41080, Spain \\ ${ }^{2}$ Institut für Physik, Universität Augsburg, Theoretische Physik I, Universitätsstraße, D-86135 Augsburg, Germany
}

(Received 23 May 2007; published 22 October 2007)

\begin{abstract}
There is an intense debate in the recent literature about the correct generalization of Maxwell's velocity distribution in special relativity. The most frequently discussed candidate distributions include the Jüttner function as well as modifications thereof. Here we report results from fully relativistic one-dimensional molecular dynamics simulations that resolve the ambiguity. The numerical evidence unequivocally favors the Jüttner distribution. Moreover, our simulations illustrate that the concept of "thermal equilibrium" extends naturally to special relativity only if a many-particle system is spatially confined. They make evident that "temperature" can be statistically defined and measured in an observer frame independent way.
\end{abstract}

DOI: 10.1103/PhysRevLett.99.170601

PACS numbers: 05.70.- a, 02.70.Ns, 03.30.+p

At the beginning of the last century, it was commonly accepted that the one-particle velocity distribution of a dilute gas in equilibrium is described by the Maxwellian probability density function (PDF)

$$
f_{M}(\mathbf{v} ; m, \beta)=[\beta m /(2 \pi)]^{d / 2} \exp \left(-\beta m \mathbf{v}^{2} / 2\right)
$$

[ $m$ : rest mass of a gas particle; v: velocity; $T=\left(k_{B} \beta\right)^{-1}$ : temperature; $k_{B}$ : Boltzmann constant; $d$ : space dimension; throughout, we adopt natural units such that the speed of light $c=1]$. When Einstein [1,2] had formulated the theory of special relativity (SR) in 1905, Planck and others noted immediately that $f_{M}$ is in conflict with the fundamental relativistic postulate that velocities cannot exceed the light speed $c$. A first solution to this problem was put forward by Jüttner [3]. Starting from a maximum entropy principle, he proposed the following relativistic generalization of Maxwell's PDF:

$$
f_{J}\left(\mathbf{v} ; m, \beta_{J}\right)=m^{d} \gamma(\mathbf{v})^{2+d} \exp \left[-\beta_{J} m \gamma(\mathbf{v})\right] / Z_{J}
$$

$\left[Z_{J}=Z_{J}\left(m, \beta_{J}, d\right): \quad\right.$ normalization $\quad$ constant; $\quad E=$ $m \gamma(\mathbf{v})=\left(m^{2}+\mathbf{p}^{2}\right)^{1 / 2}:$ relativistic particle energy; $\mathbf{p}=$ $m \mathbf{v} \gamma(\mathbf{v})$ : momentum with Lorentz factor $\gamma(\mathbf{v})=$ $\left.\left(1-\mathbf{v}^{2}\right)^{-1 / 2},|\mathbf{v}|<1\right]$. Jüttner's distribution (2) became widely accepted among theorists during the first threequarters of the 20th century [4-8] —although a rigorous microscopic derivation is lacking due to the difficulty of formulating a relativistically consistent Hamilton mechanics of interacting particles [9-13]. Doubts about the Jüttner function $f_{J}$ began to arise in the 1980s, when Horwitz et al. [14,15] proposed a "manifestly covariant" relativistic Boltzmann equation, whose stationary solution differs from Eq. (2) and, in particular, predicts a different mean energy-temperature relation in the ultrarelativistic limit $T \rightarrow \infty$ [16]. Since then, partially conflicting results and proposals from other authors [17-21] have led to an increasing confusion as to which distribution actually represents the correct generalization of the Maxwellian (1). For example, a recently discussed alternative to Eq. (2) is the "modified" Jüttner function $[18,19]$

$$
f_{M J}\left(\mathbf{v} ; m, \beta_{M J}\right)=\frac{m^{d}}{Z_{M J}} \frac{\gamma(\mathbf{v})^{2+d}}{m \gamma(\mathbf{v})} \exp \left[-\beta_{M J} m \gamma(\mathbf{v})\right] .
$$

The distribution (3) can be obtained, e.g., by combining a maximum relative entropy principle and Lorentz symmetry [20]. Compared with $f_{J}$ at the same parameter values $\beta_{J}=\beta_{M J} \lesssim 1 / m$, the modified PDF $f_{M J}$ exhibits a significantly lower particle population in the high energy tail because of the additional $1 / E$ prefactor.

Identifying the correct relativistic equilibrium velocity distribution is essential for the proper interpretation of present and future experiments in high energy and astrophysics [22-25]. Examples include the application of relativistic Langevin equations [26-28] to heavy ion collision experiments [22,25], thermalization in ultrarelativistic plasma beams [24], or the relativistic SunyaevZel'dovich (SZ) effect [23], describing the distortion of the cosmic microwave background (CMB) radiation spectrum due to the interaction of CMB photons with hot electrons in clusters of galaxies [29-31]. The predicted strength of these spectral distortions and the cosmological parameters inferred from the SZ effect depend sensitively on the assumed electron velocity distribution [23].

Relativistic molecular dynamics (MD) simulations. - To resolve the uncertainty about the relativistic equilibrium velocity PDF, we performed fully relativistic 1D MD simulations. The restriction to the 1D case is inevitable if one wants to treat localized particle interactions in a relativistically consistent manner (cf. remarks below). In our computer experiments, we simulated the dynamics of classical, impenetrable point particles with elastic pointlike binary collisions, employing an algorithm similar to those of Alder and Wainwright [32] and Masoliver and Marro [33]. The basic time step of the algorithm involves three partial tasks: (i) determine the next collision event $\left(x_{c}, t_{c}\right)$; 
(ii) evolve the system up to time $t_{c}$; (iii) calculate the momenta after the collision. The third task is solved as follows: If two particles $A$ and $B$ meet at the space-time point $\left(x_{c}, t_{c}\right)$, then they exchange momentum according to the relativistic energy momentum conservation laws $p_{A}+$ $p_{B}=\hat{p}_{A}+\hat{p}_{B}$ and $E\left(m_{A}, p_{A}\right)+E\left(m_{B}, p_{B}\right)=E\left(m_{A}, \hat{p}_{A}\right)+$ $E\left(m_{B}, \hat{p}_{B}\right)$. Given the momenta $\left(p_{A}, p_{B}\right)$ before the collision, these conservation laws determine the momenta $\left(\hat{p}_{A}, \hat{p}_{B}\right)$ after the collision by [19]

$$
\begin{aligned}
& \hat{p}_{A}=\gamma\left(v_{0}\right)^{2}\left[2 v_{0} E\left(m_{A}, p_{A}\right)-\left(1+v_{0}^{2}\right) p_{A}\right], \\
& \hat{p}_{B}=\gamma\left(v_{0}\right)^{2}\left[2 v_{0} E\left(m_{B}, p_{B}\right)-\left(1+v_{0}^{2}\right) p_{B}\right],
\end{aligned}
$$

where $v_{0}=\left(p_{A}+p_{B}\right) /\left[E\left(m_{A}, p_{A}\right)+E\left(m_{B}, p_{B}\right)\right]$ is the collision-invariant, relativistic center-of-mass velocity of the two particles. By assuming strictly localized, pointlike pair interactions, one may avoid the introduction of fields which are required when considering relativistic particle interactions at a distance (the interested reader may wish to consult the original papers of Wheeler and Feynman [9], Currie, Jordan, and Sudarshan [10], and Van Dam and Wigner [11,34], who discuss in detail the difficulties associated with classical particle-particle interactions in SR). However, considering pointlike localized interactions is expedient in the 1D case only; in higher space dimensions, the collision probability would become zero, thus preventing the system from equilibration. Moreover, if two colliding particles carry the same rest masses, then elastic 1D collisions merely interchange their velocities; hence, elastic binary collisions are not able to drive a 1D onecomponent gas to equilibrium. In our simulations, we considered a two-component mixture, consisting of $N_{1}$ light particles having equal masses $m_{1}$ and $N_{2}$ heavy particles with equal masses $m_{2}>m_{1}$. The motion of the $N=N_{1}+N_{2}$ particles was restricted to the 1D interval $[0, L]$, assumed to be stationary in the lab frame $\Sigma$. The results presented below refer to elastic reflections at the boundaries; however, we found that periodic boundary conditions yield identical outcomes if the total initial momentum was chosen to be zero in $\Sigma$. Generally, our simulations mimic a relativistic microcanonical ensemble, since the total initial energy $E_{\text {tot }}$ in $\Sigma$ is conserved in the microscopic collision processes. The above conventions define the simplest interacting model system that (i) complies with all principles of SR, (ii) does not require the introduction of interaction fields, (iii) can be simulated without further approximation, and (iv) exhibits a universal stationary equilibrium state. Hence, this model system provides an optimal test case for probing the predictions of different relativistic kinetic theories by means of numerical experiments [5-8,14,16]. Moreover, as we shall see below, it helps to clarify long-standing controversial questions regarding the definition and meaning of temperature and thermal equilibrium in SR.

Numerical results. - To identify the stationary oneparticle velocity PDFs for the light and heavy particles, respectively, we waited until the 1D two-component gas had approached the equilibrium state (typically, after $10^{2}$ collisions per particle). Then the particle velocities were measured $\Sigma$-simultaneously, i.e., at equal times with respect to the lab frame $\Sigma$. To increase the sample size, we repeated this procedure several times during a simulation run and collected the data into a single histogram. An example is shown in Fig. 1, based on a simulation with $N=10000$ particles $\left(N_{1}=N_{2}=5000, m_{2}=2 m_{1}\right)$. Each particle had been given a random initial position $x_{i}(0) \in$ $[0, L]$ and a random initial velocity $v_{i}(0)= \pm 0.8$, corresponding to a mean energy per particle $\epsilon=2.5 m_{1}$. As evident from Fig. 1, for both particle species the numerically obtained one-particle PDFs $(\diamond)$ are in very good agreement with the Jüttner function $f_{J}$ (solid line) and differ significantly from the modified distribution $f_{M J}$ (dashed lines). The same result was found for $N_{1} \neq N_{2}$.

The distribution parameters $\beta_{J / M J}$ were determined from the initial energy by means of the following consideration: If the particle numbers $N_{1}$ and $N_{2}$ are sufficiently large (thermodynamic limit), then the one-particle PDFs in the lab frame $\Sigma$ are expected to converge to either $f_{J}$ from Eq. (2) or $f_{M J}$ from Eq. (3). Generally, the mean relativistic energy value $\mu$ of a one-particle $\operatorname{PDF} f(\mathbf{v} ; m, \beta)$ is given by

$$
\mu(m, \beta)=\int_{\{|\mathbf{v}|<1\}} d^{d} \mathbf{v} f(\mathbf{v} ; m, \beta) m \gamma(\mathbf{v}) .
$$

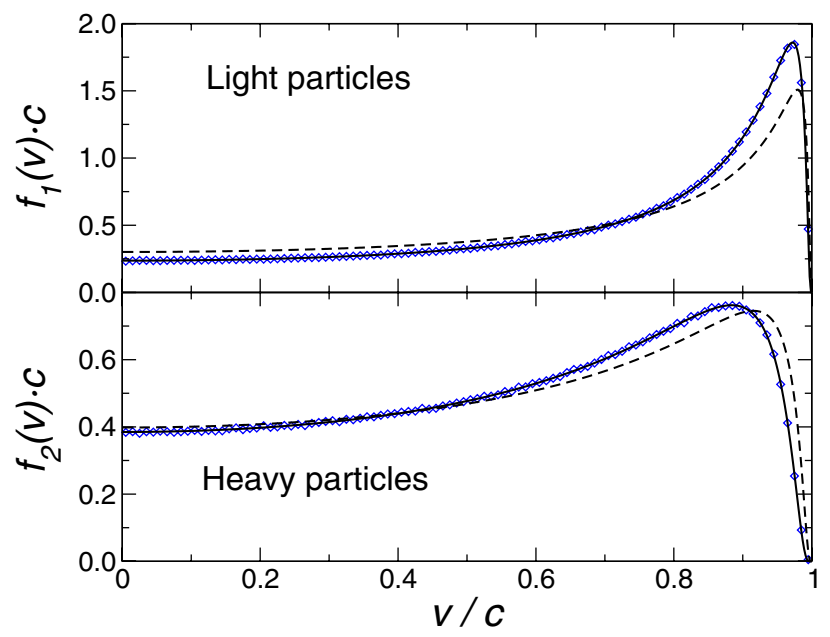

FIG. 1 (color online). Equilibrium PDFs in the lab frame $\Sigma$ : Numerically obtained one-particle velocity PDFs $(\diamond)$ based on a simulation with $N_{1}=5000$ light particles of mass $m_{1}$ and $N_{2}=$ 5000 heavy particles with mass $m_{2}=2 m_{1}$. The mean energy per particle in $\Sigma$ is $\epsilon=E_{\text {tot }} /\left(N_{1}+N_{2}\right)=2.5 m_{1} c^{2}$. The solid curves correspond to Jüttner functions (2) with the same parameter $\beta_{J}=0.702\left(m_{1} c^{2}\right)^{-1}$ but different particle masses, respectively. The dashed lines show the corresponding modified distribution (3) with $\beta_{M J}=0.402\left(m_{1} c^{2}\right)^{-1}$. As the distributions are symmetric with respect to the origin, only the positive velocity axis is shown. The simulation data are consistent with the standard Jüttner distribution (2) and thus provide evidence against the modified distribution (3). 
Assuming (i) that an equilibrium state exists where both species can be described by the same value $\beta$ and (ii) that for a gas in equilibrium the mean energy per particle is the same for particles of the same species, the total energy can be expressed as

$$
E_{\text {tot }}=N_{1} \mu\left(m_{1}, \beta\right)+N_{2} \mu\left(m_{2}, \beta\right) .
$$

In our case, the energy mean values of the two 1D candidate PDFs $f_{J}$ and $f_{M J}$ read explicitly

$$
\begin{aligned}
\mu_{J}\left(m, \beta_{J}\right) & =m\left[K_{0}\left(\beta_{J} m\right)+K_{2}\left(\beta_{J} m\right)\right] /\left[2 K_{1}\left(\beta_{J} m\right)\right], \\
\mu_{M J}\left(m, \beta_{M J}\right) & =m K_{1}\left(\beta_{M J} m\right) / K_{0}\left(\beta_{M J} m\right),
\end{aligned}
$$

with $K_{n}$ denoting modified Bessel functions of the second kind [35]. For each simulation run, the parameter tuple ( $\left.E_{\text {tot }}, N_{1}, N_{2}, m_{1}, m_{2}\right)$ is known. Hence, upon inserting them into Eqs. (6) and (7), these parameters uniquely determine the parameter value $\beta_{J / M J}$ that is consistent with the chosen velocity $\operatorname{PDF} f_{J / M J}$.

Temperature and equilibrium. - Most remarkably, in spite of the different particle masses, the two numerically obtained velocity PDFs in Fig. 1 are very well matched by Jüttner functions (2) with the same parameter $\beta_{J}$. According to our simulations, this holds true with high accuracy for a wide range of initial conditions and mass ratios. Hence, the Jüttner function not only provides the best "fit" to the numerical data, it also yields a welldefined concept of temperature in SR: Intuitively, the temperature $T$ is thought to be an intensive quantity that equilibrates to a common value if two or more systems are brought into contact with each other (i.e., may exchange different forms of energy). In our case, it is natural to consider the particle species as two different subsystems that may exchange energy via elastic collision processes. After a certain relaxation time, the combined system approaches a "thermodynamic equilibrium state," where each subsystem is described by the same asymptotic, two-parametric velocity $\operatorname{PDF} f_{J}\left(v ; m_{i}, \beta_{J}\right)$, differing only via the rest masses $m_{i}$. The commonly shared distribution parameter $\beta_{J}$ may thus be used to define a relativistic equilibrium temperature $T:=\left(k_{B} \beta_{J}\right)^{-1}$. However, for this concept to be meaningful, a restriction of the accessible spatial volume is required - be it by means of periodic boundary conditions or by imposing reflecting walls. Otherwise, it cannot be expected that a many-particle system approaches a universal stationary state which is independent of the specific initial conditions. This observation has an important implication: Any (relativistic or nonrelativistic) Boltzmann-type equation [6,8,14-16,36,37] that gives rise to a universal stationary velocity PDF implicitly assumes the presence of a spatial confinement, thus singling out a preferred frame of reference.

Moving observers and statistical thermometers. -From our simulations, we may further determine the equilibrium velocity distributions as seen from another frame $\Sigma^{\prime}$ moving with velocity $u$ relative to the lab frame $\Sigma$. Figure 2

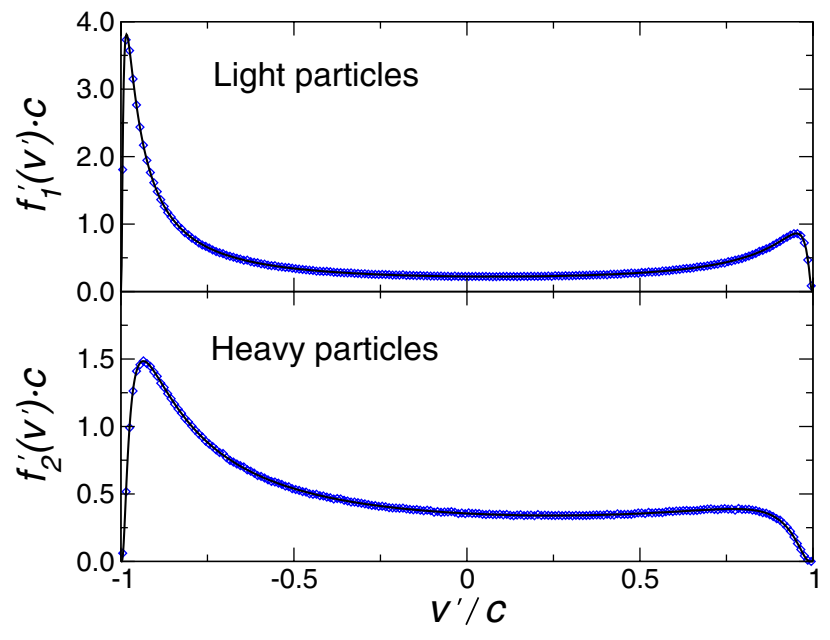

FIG. 2 (color online). Equilibrium PDFs in a moving frame $\Sigma^{\prime}$ : Velocity PDFs as measured by an observer who moves with velocity $u=0.25 c$ relative to the lab frame $\Sigma$. Parameter values are the same as in Fig. 1. The solid lines correspond to Jüttner functions $f_{J}^{\prime}$ from Eq. (8) with the same parameter $\beta_{J}=$ $0.702\left(m_{1} c^{2}\right)^{-1}$ as in Fig. 1 and different masses $m_{1}$ and $m_{2}$, respectively.

depicts the results for $u=0.25$ and the same simulation parameters as in Fig. 1. In contrast to Fig. 1, the numerical data points in Fig. 2 were obtained by measuring velocities $\Sigma^{\prime}$-simultaneously. The solid curves in Fig. 2 correspond to the PDF

$$
f_{J}^{\prime}\left(v^{\prime} ; m, \beta_{J}, u\right)=\frac{m \gamma\left(v^{\prime}\right)^{3}}{Z_{J} \gamma(u)} \exp \left[-\beta_{J} \gamma(u) m \gamma\left(v^{\prime}\right)\left(1+u v^{\prime}\right)\right]
$$

[ $v^{\prime}$ is the particle velocity in the moving frame $\left.\Sigma^{\prime}\right]$. The PDF (8) reduces to the Jüttner function (2) for $u=0 ; f_{J}^{\prime}$ is obtained by using the fact that the one-particle phase space PDF, reading

$$
\Phi_{J}(x, p)=\left(Z_{J} L\right)^{-1} \exp \left[-\beta_{J} E(m, p)\right] \Theta(x) \Theta(L-x)
$$

in $\Sigma$, is a Lorentz scalar [38,39]; $\Theta$ is the Heaviside unitstep function. Because of the excellent agreement between the numerical simulations and Eq. (8), we may state more precisely: Two relativistic gas components are in thermodynamic equilibrium for any observer if their one-particle velocity PDFs are given by generalized Jüttner functions (8) with same parameters $\beta_{J}$ and $u$. Only in this case does the net energy transfer between the different gas components in the container vanish.

Last but not least, the above results shed light on a longstanding, highly debated question [40-44] originally posed by Landsberg [45,46]:

Does a moving body appear cool? -Evidently, the answer depends on the thermometers employed by different observers. Adopting, for the reasons discussed above, $T:=$ $\left(k_{B} \beta_{J}\right)^{-1}$ as a reasonable temperature definition, a moving 
observer with rest frame $\Sigma^{\prime}$ can measure $T$ by exploiting the Lorentz invariant equipartition theorem [45]

$$
k_{B} T=m \gamma(u)^{3}\left\langle\gamma\left(v^{\prime}\right)\left(v^{\prime}+u\right)^{2}\right\rangle^{\prime},
$$

where $u=-\left\langle v^{\prime}\right\rangle^{\prime}$ and averages $\langle\cdot\rangle^{\prime}$ are taken $\Sigma^{\prime}$-simultaneously. We verified the validity of Eq. (9) explicitly by using simulation data obtained for different values of $u$. Hence, Eq. (9) defines a Lorentz invariant gas thermometer on a purely microscopic basis. Put differently, this intrinsic statistical thermometer determines the proper temperature of the gas by making use of simultaneously measured particle velocities only; thus, moving bodies appear neither hotter nor colder. Analogous considerations apply to the 2D/3D cases.

Summary. - Fully relativistic MD simulations favor the Jüttner distribution (2) as the correct relativistic oneparticle equilibrium velocity distribution. The results are conclusive for the 1D case and provide evidence against theories [14-19] that predict other distributions. Further, our simulations corroborate Landsberg's hypothesis $[45,46]$ that the temperature of classical gaseous systems can be defined and measured in a Lorentz invariant way. The extension of the MD approach to higher space dimensions is nontrivial, due to the fundamental difficulty of treating $2 \mathrm{D}$ and $3 \mathrm{D}$ two-body collisions in a relativistically consistent manner [9-13]. As a first step, it should be carefully analyzed if and how specific semirelativistic interaction models affect the $2 \mathrm{D} / 3 \mathrm{D}$ equilibrium velocity distributions.

This research was supported by the Juan de la Cierva program of the Ministerio de Ciencia y Tecnología (D. C.), the Dirección General de Enseñanza Superior of Spain by Grant No. FIS2005-02884 (J.C.-P. and D. C.), and the Junta de Andalucia (J. C.-P. and D. C.). Financial support of the German Excellence Initiative via the "Nanosystems Initiative Munich (NIM)" is gratefully acknowledged (P. H.).

[1] A. Einstein, Ann. Phys. (Leipzig) 17, 891 (1905).

[2] A. Einstein, Ann. Phys. (Leipzig) 18, 639 (1905).

[3] F. Jüttner, Ann. Phys. (Leipzig) 34, 856 (1911).

[4] W. Pauli, in Encyklöpadie der Mathematischen Wissenschaften mit Einschluss ihrer Anwendungen (Teubner, Leipzig, 1921), Vol. V/2, pp. 539-775.

[5] J.L. Synge, The Relativistic Gas (North-Holland, Amsterdam, 1957).

[6] W. Israel, J. Math. Phys. (N.Y.) 4, 1163 (1963).

[7] D. Ter Haar and H. Wegeland, Phys. Rep. 1, 31 (1971).

[8] S. R. de Groot, W. van Leeuwen, and C. G. van Weert, Relativistic Kinetic Theory: Principles and Applications (North-Holland, Amsterdam, 1980).

[9] J. A. Wheeler and R. P. Feynman, Rev. Mod. Phys. 21, 425 (1949).
[10] D. G. Currie, T. F. Jordan, and E. C. G. Sudarshan, Rev. Mod. Phys. 35, 350 (1963).

[11] H. Van Dam and E. P. Wigner, Phys. Rev. 138, B 1576 (1965).

[12] A. Komar, Phys. Rev. D 18, 1887 (1978).

[13] G. Marmo, N. Mukunda, and E. C. G. Sudarshan, Phys. Rev. D 30, 2110 (1984).

[14] L. P. Horwitz, W. C. Schieve, and C. Piron, Ann. Phys. (N.Y.) 137, 306 (1981).

[15] L. P. Horwitz, S. Shashoua, and W. C. Schieve, Physica (Amsterdam) 161A, 300 (1989).

[16] W. C. Schieve, Found. Phys. 35, 1359 (2005).

[17] G. Kaniadakis, Phys. Rev. E 66, 056125 (2002).

[18] E. Lehmann, J. Math. Phys. (N.Y.) 47, 023303 (2006).

[19] J. Dunkel and P. Hänggi, Physica (Amsterdam) 374A, 559 (2007).

[20] J. Dunkel, P. Talkner, and P. Hänggi, New J. Phys. 9, 144 (2007).

[21] R. Silva and J. A. S. Lima, Phys. Rev. E 72, 057101 (2005).

[22] H. van Hees, V. Greco, and R. Rapp, Phys. Rev. C 73, 034913 (2006).

[23] N. Itoh, Y. Kohyama, and S. Nozawa, Astrophys. J. 502, 7 (1998).

[24] M.E. Dieckmann, L. Drury, and P. K. Shukla, New J. Phys. 8, 40 (2006).

[25] R. Rapp, V. Greco, and H. van Hees, Nucl. Phys. A774, 685 (2006).

[26] F. Debbasch, K. Mallick, and J. P. Rivet, J. Stat. Phys. 88, 945 (1997).

[27] J. Dunkel and P. Hänggi, Phys. Rev. E 71, 016124 (2005).

[28] R. Zygadło, Phys. Lett. A 345, 323 (2005).

[29] R. A. Sunyaev and Y. B. Zeldovich, Comments Astrophys. Space Phys. 4, 173 (1972).

[30] M. Jones et al., Nature (London) 365, 320 (1993).

[31] M. Birkinshaw, S.F. Gull, and H. Hardebeck, Nature (London) 309, 34 (1984).

[32] B. J. Alder and T. E. Wainwright, J. Chem. Phys. 31, 459 (1959).

[33] J. Masoliver and J. Marro, J. Stat. Phys. 31, 565 (1983).

[34] H. Van Dam and E. P. Wigner, Phys. Rev. 142, 838 (1966).

[35] Handbook of Mathematical Functions, edited by $\mathrm{M}$. Abramowitz and I. A. Stegun (Dover, New York, 1972).

[36] C. Cercignani and G. M. Kremer, The Relativistic Boltzmann Equation (Birkhäuser, Berlin, 2002).

[37] H. Andŕeasson, Living Rev. Relativity 8, 2 (2005), http:// www.livingreviews.org/lrr-2005-2.

[38] N. G. van Kampen, Physica (Utrecht) 43, 244 (1969).

[39] P. A. M. Dirac, Proc. R. Soc. A 106, 581 (1924).

[40] J. H. Fremlin, Nature (London) 213, 277 (1967).

[41] I. P. Williams, Nature (London) 213, 1118 (1967).

[42] I. P. Williams, Nature (London) 214, 1105 (1967).

[43] P. T. Landsberg and G. E. A. Matsas, Phys. Lett. A 223, 401 (1996).

[44] P. D. Noerdlinger, Nature (London) 213, 1117 (1967).

[45] P. T. Landsberg, Nature (London) 214, 903 (1967).

[46] P. T. Landsberg, Nature (London) 212, 571 (1966). 